\title{
LESÕES ABDOMINAIS NOS TRAUMATIZADOS COM FRATURAS DE BACIA
}

\section{ABDOMINAL INJURIES IN PATIENTS SUSTAINING PELVIC FRACTURES}

\author{
José Gustavo Parreira, TCBC-SP ${ }^{1}$ \\ Luciana Haddad $^{2}$ \\ Samir Rasslan, TCBC-SP ${ }^{3}$
}

\begin{abstract}
RESUMO: Objetivo: Analisar as lesões abdominais e seu impacto no prognóstico dos traumatizados com fraturas de bacia. Método: Avaliamos retrospectivamente todos os prontuários das vítimas de trauma fechado com fraturas de bacia admitidos de 1996 a 2000. Nossa amostra incluiu 224 doentes, com média etária de $34 \pm$ 16 anos, sendo $137(61,1 \%)$ do sexo masculino. Dados demográficos, índices de trauma, órgãos lesados, tratamento e evolução foram estudados. As lesões abdominais foram estratificadas pela Organ Injury Scale (OIS), Abbreviated Injury Scale (AIS) e Abdominal Trauma Index (ATI). Empregamos os testes Qui quadrado e t de Student, considerando $\mathrm{p}<0,05$ como significativo. Resultados: $\mathrm{O}$ mecanismo de trauma mais comum foi o atropelamento, em 119 casos (53\%). As médias dos Revised Trauma Score (RTS) e Injury Severity Score (ISS) foram 7,0341 $\pm 1,864$ e 20,2 $\pm 12,8$. As lesões abdominais ocorreram em $95(42,4 \%)$ doentes, sendo a bexiga o órgão mais freqüentemente lesado (11\%). Sessenta e três $(28,1 \%)$ traumatizados tiveram AIS $\geq 3$ e $54(24,1 \%)$ OIS $\geq 3$ em órgãos abdominais. A média dos ATI foi $9 \pm 8$ nos com lesões abdominais diagnosticadas. Foram realizadas 55 laparotomias (nove não terapêuticas). Cinquienta e um (22\%) doentes morreram, principalmente devido ao choque hemorrágico ( 25 casos). A presença de lesões abdominais esteve relacionada significativamente com choque à admissão, fraturas complexas de bacia, fixação externa ou interna da fratura de bacia, maior morbidade e letalidade. Conclusão: As lesões abdominais são freqüentemente associadas às fraturas de bacia, e, quando presentes, relacionam-se a um pior prognóstico.
\end{abstract}

Descritores: Traumatismos abdominais: Pelve/lesões; Fraturas.

\section{INTRODUÇÃO}

Apenas 3\% de todas as fraturas ocorrem nos ossos da bacia, o que corresponde a 5\% das admissões hospitalares por trauma ${ }^{1}$. Para a rotura do anel pélvico é necessária uma colisão frontal de pelo menos $48 \mathrm{~km} / \mathrm{h}$, ou lateral de $24 \mathrm{~km} / \mathrm{h}^{2}$. Esta grande dissipação de energia é responsável pelas lesões associadas freqüientemente presentes.

A maioria das fraturas de bacia é leve e o prognóstico está mais relacionado às lesões associadas que à própria fratura ${ }^{3}$. Contudo, em alguns casos pode haver volumosa hemorragia retroperitoneal, o que eventualmente é letal ${ }^{4}$.
Nestes casos, tanto a fixação externa precoce da fratura de bacia, como a angiografia com embolização seletiva dos vasos sangrantes são métodos efetivos para a hemostasia ${ }^{5,6}$.

As lesões abdominais estão presentes em 35 a $54 \%$ dos traumatizados com fraturas de bacia, e contribuem para um aumento da letalidade em 2,3 vezes $4,7,8$. As lesões no sistema urinário, particularmente bexiga e uretra, são freqüentes, mas também são descritos traumatismos em outros órgãos que podem influenciar no prognóstico. Atualmente, a hemorragia é responsável por cerca de $30 \%$ das mortes nestes doentes, e, em 66\% das vezes, é secundária a lesões abdominais ${ }^{4}$.

1. Médico Assistente do Serviço de Emergência da Santa Casa de São Paulo.

2. Estudante de medicina da Faculdade de Ciências Médicas da Santa Casa de São Paulo.

3. Professor Titular da Disciplina de Cirurgia de Emergência da Faculdade de Ciências Médicas da Santa Casa de São Paulo. Diretor do Serviço de Emergência.

Recebido em 07/08/2001

Aceito para publicação em 26/03/2002

Trabalho realizado no Serviço de Emergência do Departamento de Cirurgia da Faculdade de Ciências Médicas da Santa Casa de São Paulo. 
Muitas vezes a identificação das possíveis lesões abdominais atrasa a fixação externa da fratura, o que pode resultar em maior perda sangüínea retroperitoneal. Nos instáveis hemodinamicamente, a lavagem peritoneal diagnóstica é o exame de escolha para avaliação abdominal em nosso meio, resultando em laparotomias não terapêuticas pela baixa especificidade do método ${ }^{1,9}$. Alguns consideram o tempo para a investigação abdominal desnecessário, mas, se não realizada, lesões importantes e hemorragia ativa intraperitoneal poderiam não ser reconhecidas.

O objetivo deste estudo é analisar a freqüência e gravidade das lesões abdominais nos traumatizados com fraturas de bacia, bem como seu impacto no prognóstico destes doentes.

\section{MÉTODO}

Este estudo foi realizado no Serviço de Emergência do Departamento de Cirurgia da Faculdade de Ciências Médicas da Santa Casa de São Paulo. Foram revisados os prontuários e protocolos de admissão de todas as vítimas de trauma fechado com fraturas de bacia admitidos de 1996 a 2000. Excluímos os casos de fraturas abertas ou patológicas e as fraturas de acetábulo isoladas, bem como os doentes que morreram na sala de admissão.

Analisamos dados demográficos, mecanismo de trauma, dados fisiológicos, Escala de Coma de Glasgow $(\mathrm{ECG})^{10}$, Revised Trauma Score (RTS) ${ }^{11}$ e órgãos lesados à admissão. As lesões abdominais foram estratificadas segundo a Organ Injury Scaling (OIS), Abbreviated Injury Scale (AIS) e o Abdominal Trauma Index (ATI) ${ }^{12-14}$. Foram levantados os métodos de avaliação diagnóstica e tratamento das lesões abdominais. Calculamos o Injury Severity Score (ISS) ${ }^{15}$ e a probabilidade de sobrevivência pelo método TRISS ${ }^{16}$. As fraturas de bacia foram classificadas conforme a proposta de Key \& Conwell, modificada por Kane ${ }^{17}$ (Tabela 1).

Tabela 1

Classificação das fraturas de bacia segundo Key \& Conwell (modificada por Kane)

\begin{tabular}{l|l}
\hline Grau & Definição \\
\hline
\end{tabular}

I Fraturas de ossos sem ruptura do anel pélvico

II Ruptura do anel pélvico em um ponto

III Ruptura do anel pélvico em mais de um ponto

IV Fraturas acetabulares

Definiu-se instabilidade hemodinâmica como pressão arterial sistólica menor que $90 \mathrm{mmHg}$. A lavagem peritoneal diagnóstica foi considerada "positiva" quando líquido hemático era recuperado do abdome. As fraturas grau III foram consideradas "complexas" para título de comparação. As laparotomias foram ditas "não terapêuticas" quando apenas a lavagem da cavidade peritoneal foi realizada. As complicações foram catalogadas conforme proposto por Hoyt et al., em $1992^{18}$. As lesões "graves" foram aquelas classificadas com AIS $\geq 3$.

Analisamos a relação entre a presença de lesões abdominais e idade, mecanismo de trauma, choque, RTS, trauma cranioencefálico, lesões torácicas, classificação de fraturas de bacia, fixação externa da fratura de bacia, AIS crânio, AIS torácico, AIS extremidades, ISS, TRISS, complicações e óbito. A relação destas variáveis com laparotomia terapêutica e ATI também foi avaliada. Empregamos os testes Qui quadrado e o teste $t$ de Student para a análise estatística univariada, considerando $\mathrm{p}<0,05$ como significativo.

Nossa amostra foi constituída por 224 doentes de dois a 78 anos, com média etária de $34 \pm 16$ anos. Cento e trinta e sete $(61,1 \%)$ eram do sexo masculino. O mecanismo de trauma mais comum foi o atropelamento, em 119 casos (53\%) (Tabela 2). Dos 224 doentes, 64 (28,6\%) foram admitidos instáveis hemodinamicamente e $33(14,7 \%)$ com escala de coma de Glasgow menor que 9. A média dos RTS à admissão foi 7,0341+1,864.

Tabela 2

Mecanismo de trauma

\begin{tabular}{lrc}
\hline Mecanismo de trauma & \multicolumn{3}{|c}{$N(\%)$} \\
\hline Atropelamento & 119 & $(53,1)$ \\
Acidentes veículos automotores & 52 & $(23,2)$ \\
Quedas & 48 & $(21,4)$ \\
Outros & 5 & $(2,2)$ \\
\hline Total & 224 & $(100)$ \\
\hline
\end{tabular}

\section{RESULTADOS}

Cento e noventa doentes $(84,8 \%)$ apresentavam alguma lesão associada, as mais freqüentes representadas pelo trauma ortopédico em extremidades, em 120 doentes (53\%). O traumatismo craniencefálico esteve presente em $78(34,8 \%)$ doentes, os órgãos torácicos foram lesados em $56(25 \%)$ e um total de 95 traumatizados $(42 \%)$ tinham lesões abdominais. Lesões graves foram encontradas no crânio em 48 (21,4\%), no tórax em $45(20,1 \%)$ e no abdome em $63(28,1 \%)$ casos. A média dos ISS da amostra foi $20,2 \pm 12,8$.

Exames complementares para avaliação de possíveis lesões abdominais foram empregados em 175 (78,1\%) doentes. A lavagem peritoneal diagnóstica foi realizada em 55 (24,5\%) casos, sendo positiva em 36 e negativa em 19. Houve dois exames falso-negativos e sete laparotomias não terapêuticas indicadas com base na positividade da LPD.

A ultra-sonografia de abdome foi empregada em 106 $(47,3 \%)$ doentes. Dos 29 exames positivos, as lesões foram constatadas por tomografia computadorizada ou la- 
parotomia em 21. Dos 77 exames negativos, houve uma lesão de bexiga intra-abdominal que necessitou de tratamento operatório posteriormente.

A tomografia abdominal computadorizada (TAC) foi realizada em 39 traumatizados $(17,4 \%)$. Houve 17 exames positivos e 22 negativos. Na evolução daqueles com "TAC normal" não houve complicações que levantassem a possibilidade de uma lesão não diagnosticada inicialmente.

As lesões abdominais estão relacionadas na Tabela 3 . $\mathrm{O}$ órgão abdominal mais freqüentemente lesado foi a bexiga, em 25 doentes (11\%). Destas, 12 eram lesões intraperitoneais, 11 extraperitoneais e 2 intra e extraperitoneais. Lesões esplênicas foram encontradas em 17 (8\%) casos, hepáticas em $16(7 \%)$ e renais em 7 (3\%). O intestino delgado foi lesado em seis $(2,7 \%)$ doentes, o cólon em sete $(3 \%)$ e o mesentério em sete (3\%). Dos 94 traumatizados com lesões abdominais, as mais graves foram classificadas como OIS grau I em 9 (9,5\%), grau II em 31 (33\%), grau III em 32 (34\%), grau IV em $16(17 \%)$ e grau V em 6 (6,4\%). A média dos ATI para toda a amostra foi $2,5 \pm 5,8$, contudo, incluindo apenas os com lesões abdominais, alcançou $9 \pm 8$. As fraturas de bacia foram classificadas como grau I em 91 (40,6\%), como grau II em 67 (30\%) e como grau III em 59 $(26,3 \%)$ casos. Dos $64(28,6 \%)$ admitidos com instabilidade hemodinâmica, $37(57,8 \%)$ apresentavam lesões abdominais (20 delas com hemorragia ativa que necessitou tratamento) e 25 (39\%) tinham fraturas complexas de bacia.

Tabela 3

Lesões abdominais

\begin{tabular}{l|cc}
\hline Órgão lesado & \multicolumn{2}{c}{$N(\%)$} \\
\hline Bexiga & 25 & $(11)$ \\
Baço & 17 & $(8)$ \\
Fígado & 16 & $(7)$ \\
Uretra & 14 & $(6)$ \\
Rim & 7 & $(3)$ \\
Cólon & 7 & $(3)$ \\
Mesentério & 7 & $(3)$ \\
Intestino delgado & 6 & $(2,7)$ \\
Vasos maiores* & 3 & $(1,3)$ \\
Diafragma & 2 & $(1)$ \\
Ovário & 1 & $(0,5)$ \\
\hline
\end{tabular}

* Vasos maiores: veia cava inferior, veias ilíaca interna ou artéria ilíaca interna.

Foram realizadas $55(24,5 \%)$ laparotomias, nove $(16,3 \%)$ delas não terapêuticas. O tratamento não operatório de lesões hepáticas, esplênicas ou renais foi indicado em seis doentes. A fixação externa ou interna das fraturas de bacia foi necessária em 50 (22,3\%), e a angiografia com embolização foi empregada em três $(1,3 \%)$ doentes.
Houve $51(22 \%)$ óbitos. A hemorragia foi a causa principal em $25(39 \%)$ casos, seguida do traumatismo cranioencefálico em $19(35 \%)$ e da sepse com falência de múltiplos órgãos e sistemas em 13 (25\%). A média dos TRISS para a amostra foi $88 \pm 25,1 \%$. Analisando apenas as 25 vítimas de hemorragia letal, notamos que 16 (64\%) possuíam lesões abdominais (12 delas com hemorragia ativa que necessitou tratamento) e $13(52 \%)$ apresentavam fraturas complexas de bacia. Dos 173 sobreviventes, 63 doentes $(36,4 \%)$ apresentaram alguma complicação, das quais sepse em $12(6,9 \%)$, choque persistente em 11 (6,3\%) e pneumonia em 11(6,3\%) foram as mais frequentes.

A presença de lesões abdominais esteve relacionada com o mecanismo de trauma (acidentes automobilísticos) $(\mathrm{p}=0,017)$, choque à admissão $(\mathrm{p}=0,005)$, fixação externa ou interna da fratura de bacia $(\mathrm{p}=0,011)$ e maior gravidade da fratura de bacia $(p=0,05)$ (Tabelas 4 e 5). Notou-se diferença significativa das médias dos ECG, AIS extremidades, RTS, ISS e TRISS entre os grupos (Tabela 6). A letalidade foi maior nos que apresentavam lesões abdominais $(\mathrm{p}=0,058)$ (Tabela 4).

As laparotomias terapêuticas foram mais frequentes nos admitidos em choque ( $\mathrm{p}=0,01)$, com fraturas complexas de bacia $(\mathrm{p}=0,025)$ e nos que necessitaram fixação da fratura de bacia $(\mathrm{p}=0,004)$ (Tabelas 5 e 7). Houve diferença significativa das médias dos ECG, AIS extremidades, RTS, ISS e TRISS entre os grupos (Tabela 8). Notou-se maior letalidade nos submetidos à laparotomia terapêutica (Tabela 7).

A presença de lesões abdominais, bem como a necessidade de laparotomia terapêutica, esteve relacionada com a gravidade da fratura de bacia (Tabela 5).

A média dos ATI foi significativamente maior nos admitidos em choque ( $\mathrm{p}<0,001)$, nos com fraturas de bacia que necessitaram fixação interna ou externa $(p=0,003)$, nos que apresentaram complicações $(\mathrm{p}=0,009)$ e nos que faleceram $(\mathrm{p}<0,001)$ (Tabela 9).

\section{DISCUSSÃO}

No início do século, a mortalidade dos traumatizados com fraturas de bacia alcançava $80 \%$, principalmente pela hemorragia retroperitoneal ${ }^{1}$. Tanto a superfície da fratura quanto a rica rede vascular presente na região são fontes de sangramento importantes. Mesmo com o conceito da reposição volêmica e evolução das medidas de suporte, a mortalidade persistia próxima a $30 \%$ na década de $1960^{1}$.

A fixação externa precoce das fraturas de bacia associada à angiografia percutânea com embolização seletiva dos vasos com sangramento ativo foi a responsável pela mudança do prognóstico deste doentes ${ }^{5-7}$. Flint et al., em 1990, relataram uma queda da letalidade em cinco vezes quando a angiografia e embolização eram empregadas ${ }^{6}$. Riemer et al., em 1993, constataram uma diminuição significativa na letalidade se a fixação externa precoce das fraturas complexas de bacia fosse realizada 5 . Atualmente, a mortalidade varia entre 7 e $23 \%$, e na nossa série alcançou $22 \%{ }^{1,3-8}$. 
Tabela 4

Comparação das variáveis nominais entre os doentes com e sem lesões abdominais

\begin{tabular}{l|c|c|c}
\hline Variável & $\begin{array}{c}\text { Lesão abdominal } \\
n=95\end{array}$ & $\begin{array}{c}\text { Sem lesão abdominal } \\
n=129\end{array}$ & $P$ \\
\hline Auto x Auto* & 30 & 22 & 0,017 \\
Choque à admissão & 37 & 27 & 0,005 \\
TCE ** & 34 & 44 & 0,9 \\
Lesão torácica & 24 & 32 & 0,9 \\
Fixação da fratura de bacia & 38 & 30 & 0,011 \\
Complicações & 32 & 31 & 0,15 \\
Óbitos & 28 & 23 & 0,058 \\
Óbito por hemorragia & 16 & 10 & 0,059 \\
\hline
\end{tabular}

* Auto x auto: automóvel versus automóvel

** TCE: Trauma cranioencefálico

Tabela 5

Presença de lesões abdominais e necessidade de laparotomia terapêutica relacionadas à gravidade da fratura de bacia

\begin{tabular}{c|c|c}
\hline Grau da fratura $(n)$ & Lesão abdominal $(\%)$ & Laparotomia terapêtica** $(\%)$ \\
\hline I $(91)$ & $31(34 \%)$ & $11(12 \%)$ \\
II $(67)$ & $29(43 \%)$ & $17(25 \%)$ \\
III (59) & $32(54 \%)$ & $17(28 \%)$ \\
\hline
\end{tabular}

$* p=0,05$

$* * p=0,025$

Tabela 6

Comparação das variáveis numéricas entre os doentes com e sem lesões abdominais

\begin{tabular}{|c|c|c|c|}
\hline & $\begin{array}{l}\text { Lesão abdominal } \\
\quad N=95\end{array}$ & $\begin{array}{c}\text { Sem lesão abdominal } \\
\quad N=129\end{array}$ & $P$ \\
\hline Idade & $33,9 \quad \pm 16$ & \pm 16 & 0,8 \\
\hline ECG & $12,3 \pm 3,8$ & $\pm \quad 3,4$ & 0,049 \\
\hline AIS crânio & $1,2 \pm 1,8$ & $1,0 \pm 1,6$ & 0,433 \\
\hline AIS tórax & $0,8 \pm 1,3$ & $\pm \quad 1,2$ & 0,55 \\
\hline AIS ext. & $3,4 \pm 0,9$ & $2,8 \pm 0,7$ & $<0,001$ \\
\hline RTS & $6,7121 \pm 11$ & $7,2712 \pm 1,3$ & 0,026 \\
\hline ISS & $28,1 \pm 11$ & $14,08 \pm 10$ & $<0,001$ \\
\hline TRISS & \pm 33 & $91,45 \pm 33$ & $<0,001$ \\
\hline
\end{tabular}

ECG: Escala de Coma de Glasgow; AIS: Abbreviated Injury Scale; RTS: Revised Trauma Score;

ISS: Injury Severity Score; TRISS: Probabilidade de sobrevivência

Em nosso serviço, o protocolo de tratamento dos traumatizados com fraturas de bacia inclui o atendimento inicial conforme proposto pelo Advanced Trauma Life Support (ATLS) ${ }^{9}$. Após a investigação radiológica inicial pertinente, a avaliação abdominal dos estáveis hemodinamicamente é realizada com ultra-sonografia ou tomografia computa- dorizada de abdome. Os instáveis hemodinamicamente são submetidos à lavagem peritoneal diagnóstica (LPD) supraumbilical. Os com fraturas graves e LPD positiva macroscopicamente são submetidos à laparotomia exploradora, seguida da fixação externa precoce da fratura de bacia, se assim indicada pelo ortopedista. Se a fixação externa preco- 
Tabela 7

Comparação das variáveis nominais entre os doentes que foram ou não submetidos à laparotomia terapêutica

\begin{tabular}{l|c|c|c}
\hline \multirow{2}{*}{ Variável } & $\begin{array}{c}\text { Laparotomia terapêtica } \\
n=45\end{array}$ & $\begin{array}{l}\text { Outros } \\
n=179\end{array}$ & $P$ \\
\hline Choque à admissão & 23 & 41 & 0,01 \\
TCE * & 18 & 60 & 0,52 \\
Lesão torácica & 15 & 21 & 0,21 \\
Fixação da fratura de bacia & 22 & 46 & 0,004 \\
Complicações & 16 & 47 & 0,29 \\
Óbitos & 21 & 30 & $<0,001$ \\
Óbito por hemorragia & 13 & 13 & $<0,001$ \\
\hline
\end{tabular}

Tabela 8

Comparação das variáveis numéricas entre os doentes que foram ou não submetidos à laparotomia terapêutica.

\begin{tabular}{l|c|rrr}
\hline & $\begin{array}{c}\text { Laparotomia terapêtica } \\
n: 45\end{array}$ & $\begin{array}{l}\text { Outros } \\
n: 179\end{array}$ & $P$ \\
\hline Idade & $33 \quad \pm 17$ & $34,3 \pm 16$ & 0,712 \\
ECG & $11,5 \quad \pm 4,2$ & $13,1 \pm 3,3$ & $<0,001$ \\
AIS crânio & 1,5 & $\pm 1,9$ & $1,0 \pm 1,6$ & 0,065 \\
AIS tórax & $1,0 \quad \pm 1,4$ & $0,6 \pm 1,2$ & 0,014 \\
AIS ext. & $3,3 \quad \pm 0,9$ & $3,0 \pm 0,9$ & $<0,001$ \\
RTS & $6,1514 \pm 2,4$ & $7,25 \pm 1,6$ & $<0,001$ \\
ISS & $30,7 \quad \pm 11,7$ & $17,3 \pm 11,0$ & $<0,001$ \\
TRISS & $64,10 \quad \pm 38$ & $92,4 \pm 19,7$ & \\
\hline
\end{tabular}

ECG: Escala de Coma de Glasgow; AIS: Abbreviated Injury Scale; RTS: Revised Trauma Score;

ISS: Injury Severity Score; TRISS: Probabilidade de sobrevivência.

Tabela 9

Comparação das médias do ATI entre os grupos com e sem fatores de risco analisados.

\begin{tabular}{l|c|c|c}
\hline Fator de risco & $\begin{array}{c}\text { Média } \pm \text { DP dos ATI } \\
\text { Fator risco presente }\end{array}$ & $\begin{array}{c}\text { Média } \pm \text { DP dos ATI } \\
\text { Fator de risco ausente }\end{array}$ & $p$ \\
\hline Choque & $5,6 \pm 9,0$ & $1,2 \pm 2,9$ & $<0,001$ \\
TCE & $3,0 \pm 6,0$ & $2,1 \pm 5,0$ & 0,25 \\
Trauma tórax & $2,8 \pm 5,6$ & $2,4 \pm 5,8$ & 0,62 \\
Fixação frat. bacia & $3,9 \pm 7,6$ & $1,5 \pm 3,9$ & 0,003 \\
Complicação & $4,1 \pm 8,3$ & $1,8 \pm 4,3$ & 0,009 \\
Óbito & $6,3 \pm 9,8$ & $1,3 \pm 3,1$ & $<0,001$ \\
\hline
\end{tabular}

TCE: trauma cranioencefálico; ATI: Abdominal Trauma Index. DP: desvio padrão.

ce não é capaz de conter a hemorragia retroperitoneal, a angiografia dos ramos da artéria ilíaca interna e a embolização seletiva dos vasos sangrantes são indicadas.

Com o controle da hemorragia retroperitoneal, as lesões associadas passaram a ser o principal fator prognóstico nos traumatizados com fraturas de bacia ${ }^{3,7}$. Cerca de
$84,8 \%$ da nossa amostra apresentava alguma lesão associada, e em porção considerável, lesões graves (AIS $\geq 3$ ). A análise da média dos índices de trauma anatômicos (ISS) também demonstra a associação de lesões em segmentos corporais distintos, denotando a gravidade destes doentes e a importância do mecanismo de trauma. Neste estudo, o 
atropelamento foi o mecanismo mais freqüentemente encontrado, o que difere das séries realizadas em outros países ${ }^{1,8}$. Alguns consideram que o atropelamento esteja relacionado com lesões orgânicas mais graves, o que determinaria maior letalidade ${ }^{19,20}$.

Nossos dados constatam que a maioria das fraturas de bacia e leve e não necessita de tratamento específico, fato já relatado em outras séries 1,8,20,21. Contudo, as fraturas foram classificadas como complexas em $26,3 \%$, a necessidade de fixação ocorreu em 22,3\%, e a angiografia com embolização foi empregada em $1,3 \%$ dos casos. Portanto, uma parcela considerável destes doentes necessita atenção específica ao tratamento da fratura de bacia, caso contrário um aumento na mortalidade e complicações devem ser esperados.

Neste grupo de traumatizados com fraturas de bacia, as lesões abdominais foram freqüentemente encontradas, o que justifica a investigação ativa em todos estes casos. A ultra-sonografia de abdome foi o método diagnóstico mais empregado $(47,3 \%)$, contudo houve limitação quanto à análise dos seus resultados. Exames positivos não tiveram as lesões confirmadas por laparotomia ou tomografia computadorizada em $27 \%$ e exames negativos não foram capazes de excluir totalmente a presença de lesões abdominais em $1 \%$. Considera-se que, com o treinamento adequado, este exame oferece uma sensibilidade de até $90 \%$ para detecção de hemoperitônio $^{22}$. Já a tomografia computadorizada demonstrou maior credibilidade nos resultados, mesmo que tenha sido realizada apenas em 17,4\% dos casos. Nos últimos anos, a tomografia computadorizada espiral ou helicoidal se estabeleceu como padrão para a avaliação abdominal. É pouco invasiva, com altos índices de sensibilidade e especificidade ${ }^{23}$.

Devido ao custo envolvido, parece razoável a utilização da ultra-sonografia como exame de triagem, e, em casos selecionados, a tomografia estaria indicada. Caso a fratura seja complexa, a TAC é o exame de escolha, não somente para avaliação abdominal mais detalhada, mas também para a análise da própria fratura. $\mathrm{O}$ emprego de métodos de imagem é a regra nos estáveis hemodinamicamente, pois há a possibilidade de tratamento não operatório de lesões em vísceras abdominais como baço, fígado e rim ${ }^{24}$. Estes órgãos foram lesados em $18 \%$ da nossa amostra, e o tratamento não operatório foi possível em seis doentes. É importante a avaliação da bexiga através de cistografia na suspeita de lesão extra ou intraperitoneal, vista a falha do ultra-som em detectar uma lesão intraperitoneal que necessitou de tratamento operatório posteriormente.

Nos instáveis hemodinamicamente, a lavagem peritoneal diagnóstica foi empregada determinando sete laparotomias não terapêuticas em 55 (12,7\%). Houve também falso-negativos em 2,6\%. A baixa especificidade deste método diagnóstico é relatada, mas devido à impossibilidade de remoção destes doentes para realização de exames no departamento de radiologia, a LPD continua sendo o método de escolha em nosso meio ${ }^{1,4,21}$. Aumenta o interesse no exame ultra-sonográfico realizado na sala de emergência pelo próprio cirurgião, denominado Focused Assessment Sonography for Trauma (FAST) ${ }^{25}$. Este é um método promissor que ganhou campo nos cenários norteamericanos na última década. Permite a avaliação da presença de líquido livre intraperitoneal, e, em situações de instabilidade hemodinâmica, pode auxiliar na indicação ou não de laparotomia sem a necessidade da lavagem peritoneal diagnóstica.

As lesões de bexiga e uretra foram observadas em $16 \%$. Deve-se sempre suspeitar da presença de lesões em sistema urinário frente a traumatizados com fraturas de bacia, indicando-se os exames complementares (uretrografia, cistografia) para o diagnóstico, visto que a maioria destes doentes necessita tratamento operatório ${ }^{26,27}$.

As lesões intestinais por trauma fechado não são freqüentes, entretanto há dificuldade para o seu diagnóstico diante das limitações dos exames de imagem na sua detecção ${ }^{28}$. A dor desencadeada pela própria fratura de bacia dificulta ainda mais o exame físico. Há, portanto, condições propícias para a falha na identificação destas lesões, determinando conseqüências graves, até mesmo óbito ${ }^{28}$. Em nosso estudo, as lesões de vísceras ocas foram encontradas em 6\%. Mesmo não sendo tão freqüentes, consideramos importante manter a atenção voltada também para estas lesões, devido às complicações do diagnóstico tardio.

$\mathrm{Na}$ avaliação da gravidade das lesões abdominais, notamos que a maioria foi classificada como OIS II (33\%) e III (34\%). Lesões graves (AIS $\geq 3$ ) foram encontradas em $28 \%$ da amostra. Nos que apresentavam traumatismo abdominal, a média dos ATI foi $9 \pm 8$, o que ratifica a gravidade das lesões abdominais nos traumatizados com fraturas de bacia. Cerca de 24,5\% dos doentes foram submetidos à laparotomia exploradora, e outros seis ao tratamento não operatório de fígado, baço ou rim. Ou seja, as lesões abdominais são freqüentes e necessitam de tratamento específico muitas vezes.

A hemorragia foi a causa principal de óbito, seguida do trauma cranioencefálico e sepse. Lesões abdominais foram foco de sangramento em $64 \%$ das vítimas de hemorragia letal, em geral associadas a fraturas complexas de bacia. Não houve óbitos por sepse decorrente de lesões abdominais. A letalidade da amostra (22\%) é alta, contudo a média das probabilidades de sobrevivência calculada foi $88 \pm 25,1 \%$.

A análise estatística demonstrou maior frequiência de lesões abdominais e necessidade de laparotomia terapêutica nos admitidos em choque, nos portadores de fraturas complexas de bacia e nos que necessitaram fixação das fraturas de bacia. Notou-se também que os índices de trauma e cálculo de probabilidade de sobrevivência demonstraram que os portadores das lesões abdominais eram mais graves e com pior prognóstico. A mesma tendência foi observada com a avaliação e comparação das médias dos ATI entre os com e sem os fatores de risco anteriormente citados.

Portanto, há uma associação de choque, lesões abdominais de possível tratamento operatório e fraturas complexas de bacia, o que determina pior prognóstico. Bond 
et al. também relataram associação da gravidade das fraturas de bacia e a presença de lesões abdominais ${ }^{29}$.

O prognóstico das vítimas de fraturas de bacia parece estar relacionado na maioria das vezes às lesões associadas, visto que freqüentemente as fraturas são leves e não determinam grande hemorragia ${ }^{3,7}$. Contudo, mesmo nos com fraturas complexas, parece haver grande influência das lesões associadas no prognóstico, especialmente as abdominais. Atualmente as séries publicadas confirmam a hipótese de que as fraturas de bacia isoladamente são responsáveis apenas por uma pequena porcentagem dos óbitos nestes doentes ${ }^{30}$.

Uma das situações mais difíceis na prática clínica é o tratamento de politraumatizados admitidos instáveis hemodinamicamente e que apresentam fraturas complexas de bacia. Importante neste momento, além da reposição volêmica, é detectar e controlar o foco de sangramento. Os dados destes estudo sugerem que a avaliação abdominal com LPD ou FAST seja a prioridade, seguida de laparotomia exploradora, fixação externa da fratura e angiografia se necessário. Nestes momentos iniciais, algum método incruento para a fixação da fratura de bacia pode ser empregado. Se disponível, equipamentos para fixação externa posterior que podem ser empregados na sala de emergência como o C-clamp são uma opção ${ }^{1,21}$. Na positividade da LPD, a laparotomia está indicada. Caso não se constate presença de lesões intracavitárias com sangramento ativo e haja um grande hematoma retroperitoneal pélvico, é prudente tamponar a pelve com compressas, fechar temporariamente o abdome e realizar a fixação externa da fratura da bacia antes do tratamento definitivo das lesões abdominais. Após o término deste procedimento, a cavidade peritoneal é explorada novamente e o tratamento definitivo realizado.

$\mathrm{Na}$ presença de acidemia, hipotermia, coagulopatia persistentes, técnicas para controle do dano podem ser empregadas. A operação é interrompida após o controle do sangramento proveniente de grandes vasos e vísceras maciças, outras lesões têm o tratamento definitivo postergado. Após a síntese temporária do abdome, o doente é encaminhado à unidade de tratamento intensivo e a relaparotomia programada. Também deve-se ressaltar que o atendimento de casos graves é multidiciplinar, com participação não só do cirurgião mas também do ortopedista já nos primeiros momentos.

Assim, a análise desta casuística mostra que as lesões abdominais são freqüentes nos traumatizados com fraturas de bacia e sua presença determina pior prognóstico. Estas lesões estão associadas ao choque hemorrágico à admissão e fraturas complexas de bacia, e, muitas vezes, necessitam de tratamento operatório.

\begin{abstract}
Background: To analyze possible abdominal injuries and their impact on the prognosis of patients sustaining pelvic fractures. Method: Retrospective chart review including all victims of pelvic fractures due to blunt trauma from 1996 until 2000. Sample was constituted of 224 patients, mean age $34 \pm 16$ years, 137 (61,1\%) male. Demographic data, trauma indices, organs injured, treatment and evolution were assessed. Abdominal injuries were graded using Organ Injury Scale (OIS), Abbreviated Injury Scale (AIS) and Abdominal Trauma Index (ATI). Statistical analysis was carried out with student's t and chi square tests. Results: Pedestrian struck was the most common mechanism of injury (53\%). Mean Revised Trauma Score (RTS) and Injury Severity Score (ISS) were $7.0341 \pm 1.864$ and $20.2 \pm 12.8$ respectively. Injuries in abdominal organs were found in 95 patients $(42,4 \%)$, being the bladder the most frequently injured $(11 \%)$. Sixty three $(28,1 \%)$ patients had their abdominal injuries graded as $A I S \geq 3$, and 54 (24,1\%), as OIS $\geq 3$. Mean ATI for those sustaining abdominal lesions was $9 \pm 8$. Fifty five laparotomies were performed, nine of them being non therapeutic. Fifty one patients died (22\%), mainly due to hemorrhagic shock. The presence of injuries to abdominal organs were significantly associated with shock upon admission, complex pelvic fractures, external fixation of the pelvic fracture, increased number of complications and higher mortality $(p<0.05)$. Conclusion: Abdominal organs are frequently injured in patients sustaining pelvic fractures and, when present, determine worse prognosis.
\end{abstract}

Keywords: Abdominal injuries; Pelvis/injuries; Fractures.

\section{REFERÊNCIAS}

1. Cryer HG, Johnson EE. Pelvic Fratures. In Feliciano D, Moore EE, Mattox K. Trauma - $3^{\text {rd }}$ ediction. Stamford, Connecticut. Appleton and Lange, 1996, pp 635 - 661.

2. McCoy GF, Johnstone RA, Kenright J. Biomechanical aspects of pelvic and hip injures in road traffic accidents. J Orthop Trauma 1989; 3: 118-123.
3. Parreira JG, Coimbra R, Rasslam $\mathrm{S}$ et al. The role of associated injuries on outcome of blunt trauma patients sustaining pelvic fractures. Injury, 2000, 31: 677-682.

4. Parreira JG, Coimbra R, Silva LE et al. A critical analysis of prognostic factors in patients sustaining pelvic fractures admitted in shock. Panam J Trauma,1998, 7: 2733. 
5. Bassan D, Cephas GA, Fergusson KA et al. A protocol for the initial management of unstable pelvic fractures. Am Surg, 1998, 64: 862-867.

6. Wong YC, Wang LJ, Ng CJ et al. Mortality after successful transcatheter arterial embolization in patients with unstable pelvic fractures: rate of blood transfusion as a predictive factor. J Trauma, 2000, 49: 71-74.

7. Poole G, Ward EF, Muakassa FF et al. Pelvic fracture from major blunt trauma. Outcome is determined by associated injuries. Ann Surg, 1991; 213: 532 - 538.

8. Burguess AR, Eastridge BJ, Young JWR et al. Pelvic ring disruptions: effective classification system and treatment protocols. J Trauma, 1990, 30: 848-856.

9. American College of Surgeons Commitee on Trauma: Advenced Trauma Life Support For Doctors - Instructor Course Manual. Chicago, American College of Surgeons, 1997, 990p.

10. Teasdale G \& Jennet B. Assessment of coma and impaired consciuosness: a pratical scale. Lancet, 1974, 2: 8184.

11. Champion HR, Sacco WJ, Copes W et al. A revision of the trauma score. J Trauma 1989; 29(5): 623 - 629.

12. Moore EE, Cogbill T, Malangoni M et al. Organ Injury Scaling. Surg Clin N Am 1995; 2: 293 - 303.

13. The Abbreviated Injury Scale. 1990 Revision. Association for Advancement of Automotive Medicine. 1990, Des Plaines, IL 60018, USA.

14. Moore EE, Dunn EL, Moore J et al. Penetrating abdominal trauma index. J Trauma, 21: 439-445, 1981.

15. Baker S, O'Neil B, Haddon W et al. The Injury Severity Score: A method for describing patients with multiple injuries and evaluating emergency care. J Trauma, 1974, 14: 187-196.

16. Boyd CR, Tolson MA, Copes WS. Evaluating trauma care: The TRISS method. J Trauma, 1987, 27: 370 - 377.

17. Kane WJ. Fractures of the Pelvis. In Rochwood CA, Green DP: Fractures. Philadelphia, JB Lippincott, 1984, pp. 1093.

18. Hoyt DB, Fridlund PH, Fortlage D et al. - Evaluation of provider-related and disease-related morbidity in a level I university trauma service: directions for quality improvment. J Trauma,1992, 33: 586 - 601.

19. Rothenberger D, Fischer R, Strate R et al The mortality associated with pelvic fractures. Surgery, 1978, 84: 356-361.

20. Eastridge B, Burgess A. Pedestrian pelvic fractures: 5-year experience of a major urban trauma center. J. Trauma, 1997, 42: 695 - 700 .

21. Scalea T, Burgess A. Pelvic Fratures. In Mattox K, Feliciano D, Moore EE. Trauma $-4^{\text {th }}$ ediction. United States of America. McGraw-Hill, 2000, pp 807-836.

22. Boulanger B, Rozycky G, Rodriguez A. Avaliação ultrassonográfica da lesão traumática. Clin Cir Am Norte, 1999, 79: 1227-1246.
23. Nance M, Peden G, Shapiro M et al. Solid viscus injury predicts major viscus in blunt abdominal trauma. $\mathbf{J}$ Trauma, 1997, 43: 618-623.

24. Knudson M, Maull K. Tratamento não cirúrgico (conservador) das lesões em órgãos sólidos: Passado, presente e futuro. Clin Cir Am N, 1999, 79:1289-1303.

25. Rozycki GS \& Shackford S. Ultrasound, what every trauma surgeon should know. J Trauma, 1996, 40: 1-4.

26. Rehn C, Mure A, Malley K et al. Blunt traumatic bladder rupture: the role of retrograde cystogram. Ann Em Med, 1991, 20: 845 - 847.

27. Koraitin M. Pelvic fracture urethral injuries: the unresolved controversy. J. Urol, 1999, 161: 1433-1441.

28. Allen G, Moore F, Cox C et al. Hollow visceral injury and blunt trauma. J Trauma, 1998, 45: 69-78.

29. Bond S, Gotschall C, Eichelberger M. Predictors of abdominal injury un children whit pelvic fracture. J. Trauma, 1991, 31: 1169-1173.

30. Rittmeister M, Lindsey R, Kohl. Pelvic fracture among polytrauma decedents. Trauma-based mortality with pelvic fracture - a case of 74 patients. Arch Orthop. Trauma Surg, 2001, 121: 43-49.

Endereço para correspondência:

José Gustavo Parreira

Rua Dona Veridiana 167 - ap. 83

01238-010 — São Paulo-SP

Email: jgparreira@uol.com.br 